\title{
ESTUDIANTES UNIVERSITARIOS CON TRASTORNOS DEL ESPECTRO DEL AUTISMO: REVISIÓN DE SUS NECESIDADES Y NOTAS PARA SU ATENCIÓN
}

\section{College Students with Autism Spectrum Disorders: Review of their Needs and Notes for their Attention}

\author{
Francisco Alcantud Marín \\ Universitat de Valencia. Facultad de Psicología \\ Departamento de Psicología Evolutiva y de la Educación \\ Francisco.Alcantud@uv.es \\ Yurena Alonso-Esteban \\ Universitat de Valencia. Facultad de Psicología \\ Departamento de Psicología Evolutiva y de la Educación
}

Recepción: 6 de febrero de 2020

Aceptación: 6 de noviembre de 2020

Resumen: En los últimos 10 años hemos visto incrementar el número de publicaciones relacionadas con la inclusión en las aulas universitarias de estudiantes con Trastornos del Espectro del Autismo, síndrome de Asperger o Autismo de alto funcionamiento. En este artículo, se resume la literatura sobre la presencia de estudiantes con Trastornos del Espectro del Autismo en la Universidad y se reflexiona sobre la necesidad de desarrollar programas específicos para su atención en el marco de los servicios de apoyo a los estudiantes con discapacidad u otras estructuras de servicios similares. Entre las medidas específicas, hemos encontrado experiencias sobre programas de sensibilización a profesores y estudiantes con la finalidad de generar una cultura sobre "el autismo", programas para tratar la ansiedad, programas de prevención de salud mental, etc. En definitiva, medidas que, aunque están dirigidas a un colectivo en particular, pueden beneficiar a toda la comunidad universitaria.

Palabras clave: Trastornos del Espectro del Autismo; acceso a la Universidad; medidas de inclusión. 
AвSTRACT: In the last 10 years we have seen an increase in the number of publications related to the inclusion in university classrooms of students with Autism Spectrum Disorders, Asperger's Syndrome or high functioning autism. This article summarizes the literature on the presence of students with Autism Spectrum Disorders at the University and reflects on the need to develop specific programs for their care within the framework of support services for students with disabilities or other similar service structures. Among the specific measures, we have found experiences on awareness programs for teachers and students with the aim of generating a culture about "autism", programs to treat anxiety, mental health prevention programs, etc. In short, measures that, although they are aimed at a particular group, can benefit the entire university community.

KeYwords: Autism Spectrum Disorders; college access; measures of inclusion.

\section{Introducción}

4

N LOS Últimos 25 AÑos, son evidentes los avances realizados en las universidades españolas en accesibilidad, equiparación de oportunidades y desarrollo de

programas psicoeducativos para el apoyo a estudiantes con discapacidad, sobre todo a partir de la publicación y entrada en vigor de la Ley 51/2003 de Igualdad de Oportunidades, no Discriminación y Accesibilidad Universal $(B O E, 2003)$ y de la Ley Orgánica 4/2007 de Universidades (BOE, 2007). En ambas normas, se entiende por discapacidad la reconocida por los órganos competentes, y no las necesidades educativas específicas, lo que en principio genera una cierta falta de armonía respecto a otros países y respecto a los apoyos recibidos por los estudiantes en los ciclos formativos previos. En el caso de las universidades españolas, las categorías contempladas se refieren a Discapacidad Física (sea orgánica o motriz), Sensorial (visual, auditiva o ambas), Psíquica (trastornos mentales, discapacidad intelectual o del desarrollo) y un último tipo que se denomina "Otros tipos de discapacidad" (Universia, 2016). En la actualidad, se estima que entre el 1,5-1,7 \% de los estudiantes matriculados en las universidades españolas declaran padecer algún tipo de discapacidad (Universia, 2016, 2018). De ellos, el 14,8 \% se clasifican en la categoría de Discapacidad Psíquica. Es de destacar que el 25,6 \% lo hacen en la categoría de "Otros tipos de discapacidad" (Universia, 2016).

Los estudiantes con Trastornos del Espectro del Autismo, síndrome de Asperger o Autismo de alto funcionamiento podrían estar incluidos en cualquiera de las dos categorías (Discapacidad Psíquica u Otros tipos de discapacidad). El hecho de incluirlos en una categoría denominada Discapacidad Psíquica junto a los trastornos mentales o en “otras discapacidades" hace pensar en el desconocimiento de la naturaleza de este trastorno, o quizás los propios estudiantes voluntariamente solicitan ser incluidos en "otras discapacidades", o tal vez rehúyan una etiqueta diagnóstica que conste en su expediente, por temor al rechazo, que seguramente ya han experimentado en otras ocasiones. En este artículo, se quiere analizar, desde un punto de vista teórico y documentado, los condicionantes de los estudiantes universitarios que presentan un Trastorno del Espectro del Autismo y las soluciones propuestas para su inclusión y mejora de su calidad de vida durante su tránsito por las aulas universitarias. 
ESTUDIANTES UNIVERSITARIOS CON TRASTORNOS DEL ESPECTRO DEL AUTISMO: REVISIÓN DE SUS NECESIDADES Y NOTAS PARA SU ATENCIÓN FRANCISCO ALCANTUD MARÍN Y YURENA ALONSO-ESTEBAN

\section{Trastornos del Espectro del Autismo}

Los Trastornos del Espectro del Autismo (TEA) son trastornos del neurodesarrollo que se caracterizan por déficits persistentes en la comunicación e interacción social y patrones repetitivos y restringidos de conductas, actividades e intereses que causan alteraciones significativas en su desarrollo a nivel social, laboral o en otras áreas del funcionamiento (APA, 2013). Con la publicación del DSM-5 (APA, 2013) las categorías diagnósticas de Trastornos Generalizados del Desarrollo (TGD) del DSM-IV (APA, 1994), como son los TGD no específicos, síndrome de Asperger y Trastornos Autistas, quedaron subsumidas bajo la categoría de TEA.

Según los últimos datos publicados por el $\mathrm{CDC}^{1}$, la prevalencia en USA es de 1/59 niños y niñas (Baio et al., 2018). Aunque existen múltiples publicaciones que cuestionan por razones metodológicas estas cifras, la inmensa mayoría confirman un incremento de la incidencia de los TEA en las últimas décadas (Alcantud-Marín et al., 2016). Entre las razones que justifican el incremento en las tasas de TEA, destacan los cambios de criterios diagnósticos, el mayor conocimiento y capacidad de detección, y una cierta permuta de diagnóstico (Fombonne, 2009; King y Bearman, 2009). Parece que, mientras que los índices de Discapacidad Intelectual descienden, los índices de TEA aumentan (Shattuck, 2006). Otras razones pueden ser el incremento en el diagnóstico de TEA de alto funcionamiento (Braun et al., 2015). En nuestro país, los pocos estudios sobre la prevalencia del trastorno de los que disponemos apuntan la misma tendencia que los del CDC, aunque con una incidencia y prevalencia más baja (Canal et al., 2010; Fortea et al., 2013; Morales-Hidalgo et al., 2018; Pérez-Crespo et al., 2019).

Aunque los TEA se consideran como graves, crónicos y persistentes a lo largo de todo el ciclo vital, desde ya hace algún tiempo se observa como un porcentaje de estos casos alcanzan niveles intelectuales y habilidades sociales como el promedio de la población. En los primeros trabajos de seguimiento de Rutter (1970), se evidenciaba que un número de casos diagnosticados como TEA $(1,5 \%)$ alcanzaron niveles de funcionamiento normal en la edad adulta. En un estudio de seguimiento, treinta años después, Sigman y colaboradores (1999) elevan el porcentaje de "normalización o recuperación” hasta el $17 \%$. Los últimos estudios consultados (Helt et al., 2008) concluyen que entre el $3 \%$ y el $25 \%$ de las personas inicialmente diagnosticadas como TEA en la edad adulta presentan competencias en áreas sociales y comunicativas semejantes a las de desarrollo típico. Todo hace pensar que, en algunos casos, y como consecuencia de la intervención o menor grado de trastorno neurológico de base, los síntomas de los TEA se atenúan. Billstedt et al. (2007) hacen un estudio longitudinal después del primer diagnóstico (entre 13 y 22 años), llegando a la conclusión de que los problemas de interacción social persistían en la mayoría de los adultos, mientras que los problemas de conducta restrictiva eran mucho más variables. En este sentido, apuntamos que, aunque como consecuencia de la intervención los síntomas nucleares puedan atenuarse, enmascararse e incluso llegar

1 CDC: Center for Disease Control and Prevention (https://www.cdc.gov/).

Ediciones Universidad de Salamanca / CC BY-NC-ND

Siglo Cero, vol. 52 (2), 2021, abril-junio, pp. 83-100 
a desaparecer, si las demandas de interacción social se incrementan o desaparecen los apoyos, pueden reproducirse los síntomas nucleares de los TEA e incluso agravarse con la aparición de otros trastornos comórbidos (Watkins et al., 2019).

A nivel individual, los estudiantes con TEA pueden tener diferentes problemas relacionados con los déficits nucleares del trastorno.

- Déficit en la función ejecutiva: la función ejecutiva es un constructo complejo y las personas con TEA pueden tener dificultades en diferentes aspectos de esta función. Por ejemplo, a la hora de planificar y organizar el estudio, lo que puede conducirle al fracaso cuando la tarea propuesta se descompone en etapas o fases; a la hora de adaptarse a los posibles cambios en la rutina, por su falta de flexibilidad, por ejemplo, exigiendo sentarse siempre en el mismo lugar en el aula o en la cafetería. También pueden presentar dificultades de memoria o de orientación a la hora de desplazarse por el campus universitario. Pueden presentar dificultades a la hora de mantener la atención o la concentración en el trabajo independiente, etc. Esta función también les dificultará la participación en debates donde se contrapongan ideas (Berger et al. et al., 2003; Jansen et al. et al., 2017).

- Coherencia central débil: los estudiantes con TEA pueden presentar problemas en relación a la conceptualización global o desarrollo de ideas síntesis debido al déficit de coherencia central (Berger et al. et al., 2003). Sin embargo, este mismo punto débil, en ocasiones y según qué tarea, puede convertirse en un punto fuerte.

- Comunicación: se informa que los estudiantes con TEA pueden tener problemas de comunicación verbal y no verbal. Como aún la estrategia de enseñanza básica en la Universidad es la clase magistral, esto puede generar en los estudiantes problemas de comprensión en el mensaje del profesorado. Por ejemplo, pueden hacer la misma pregunta varias veces al no comprender conceptos abstractos (Jansen et al. et al., 2017; Zürcher et al., 2013). En este sentido, los estudiantes prefieren el uso de sistemas de aprendizaje combinado (Blended Learning) o contenidos “on line” (Satterfield et al. et al., 2015).

- Interacción social: los problemas de comunicación, fijación de la mirada, además de la experiencia aprendida, hacen que los estudiantes con TEA tengan dificultades a la hora de relacionarse con otras personas (con sus iguales, personal de administración o profesorado) (Berger et al. et al., 2003; Orsmond et al. et al., 2013).

- Intereses restringidos: las personas con TEA pueden presentar conductas repetitivas o intereses restringidos en un área en particular. Los estudiantes con TEA en los que se combine este interés con el área académica podrán dedicar mucho más tiempo a la tarea universitaria y como consecuencia alcanzar resultados brillantes.

- Otros problemas asociados: los tics o movimientos estereotipados, hipersensibilidad sensorial o autorregulación de la ansiedad pueden presentarse también como problemas en los estudiantes con TEA (Jansen et al. et al., 2017). 
Las dificultades de los estudiantes universitarios con TEA están en línea con los que presentan todas las personas con este trastorno (Billstedt et al. et al., 2007). Según encuestas (Harpur et al. et al., 2004), muchas personas con TEA se sienten marginadas, son víctimas de acoso, desarrollan complejos de inferioridad y desarrollan con frecuencia sentimientos de frustración que, por sus dificultades en la regulación emocional, pueden hacer aparecer otros trastornos en forma de crisis. De acuerdo con Bellini $(2004,2006)$ y como consecuencias de este mal ajuste, las personas con TEA pueden desarrollar ansiedad social, que puede llegar a fobia social y, como medida de evitación, el aislamiento social. Algunos resultados empíricos, además, apuntan en la dirección de que son las personas con TEA de alto funcionamiento las que presentan, con mayor frecuencia e intensidad, trastornos de ansiedad (Davis et al., 2011). La investigación demuestra que a los estudiantes con TEA les cuesta un mayor esfuerzo alcanzar el grado que a sus compañeros neurotípicos, presentando a su vez un alto nivel de soledad, ansiedad y depresión, con una alta tasa de abandono antes de alcanzar la graduación (Jackson et al. et al., 2018).

Dadas las dificultades que presentan estos estudiantes, parece obvio que necesitan adaptaciones específicas para poder desarrollar su función de forma equitativa con el resto de estudiantes (Gelbar et al. et al., 2015; Jansen et al., 2017; Van Hees et al. et al., 2015).

\section{Prevalencia de estudiantes con TEA en la Universidad}

Como consecuencia del incremento de la prevalencia del trastorno, las universidades se enfrentan cada día a un mayor número de estudiantes con TEA (Grogan, 2015). En el estudio NLTS 2 (National Longitudinal Transition Study 2: Newman et al., 2011) del Departamento de Educación de USA, se analizó la trayectoria y transición de una muestra de estudiantes que tenían entre 13 y 16 años en el año 2000, hasta el año 2009 (21 a 25 años de edad al finalizar la recogida de datos). Respecto a los estudiantes que tenían diagnóstico de TEA se observó que solo el $1 \%$ de los mismos alcanzaron los estudios universitarios.

Otros estudios apuntan que cada año en USA terminan el High School ${ }^{2}$ unos 49000 estudiantes con diagnóstico de TEA. De ellos aproximadamente 16000 (32,65 \%) siguen estudios universitarios (Wei et al. et al., 2016). White y cols. (2011) llegan a analizar la población universitaria mediante la escala Ritvo (Ritvo Autism Asperger Diagnostic Scale-Revised: Ritvo et al., 2011), llegando a concluir que "entre el 0,7 y el $1.9 \%$ de los estudiantes universitarios podrían cumplir los criterios diagnósticos de TEA" (White et al. et al., 2011, p. 683).

En el marco de los estudiantes con discapacidad, sin embargo, Wei et al. (2013) establecen que los estudiantes con TEA tienen la tercera tasa más baja entre las 11 categorías de estudiantes con discapacidad que ellos contemplan. Otra realidad es que, aunque los estudiantes con TEA acceden a la Universidad, solo el 38,8\% de ellos

2 High School: el grado en el High School equivale al Bachiller.

Ediciones Universidad de Salamanca / CC BY-NC-ND

Siglo Cero, vol. 52 (2), 2021, abril-junio, pp. 83-100 
llegan a graduarse (Newman et al., 2011). El alto abandono de los estudios universitarios se justifica, en gran medida, por la falta de apoyos (VanBergeijk et al.et al., 2008). En España, no disponemos de información estadística sobre el número de estudiantes con TEA que acceden a la Universidad, aunque sí disponemos de evidencias de su existencia (Aguilar-Pérez, 2011; De la Fuente-Anuncibay y Cuesta-Gómez, 2017; Vilalta-Porta et al. et al., 2018; Valdunquillo-Carlón e Iglesias-Rodríguez, 2015).

\section{Servicios de apoyo a estudiantes con TEA en la Universidad}

La problemática de los estudiantes con TEA en la Universidad está siendo objeto de estudio, hasta el punto de que ya se han realizado algunas revisiones sistemáticas de la literatura (Anderson et al. et al., 2017; Gelbar et al. et al., 2014). En la primera, Gelbar et al. (2014), se revisaron 18 estudios que describen la experiencia de 68 estudiantes universitarios con TEA. En la segunda, Anderson et al. (2017) analizan veintitrés estudios, de los veintinueve encontrados con datos sobre los beneficios, desafíos y barreras encontradas en su acceso y tránsito por la Universidad. Ambas revisiones ponen de relieve las dificultades a niveles sociales, emocionales y sensoriales que presentan los estudiantes con TEA y cómo estas dificultades impactan negativamente en sus resultados.

Los servicios de apoyo a estudiantes con discapacidad, desafortunadamente, no han desarrollado actividades específicas para atender las complejas necesidades de los estudiantes con TEA (Hendricks y Wehman, 2009); los apoyos ofrecidos son, con frecuencia, incompatibles con la necesidad. Los problemas de los estudiantes con TEA de los que se ha encontrado alguna información se centran en las dificultades que tienen a la hora de resolver problemas sociales y académicos (Anderson et al. et al., 2017; Gurbuz et al. et al., 2019; Jackson et al. et al., 2018; Sarrett, 2018). Un segundo grupo de publicaciones hacen referencia a los problemas relacionados con la autorregulación emocional y cambio de estado de ánimo (estrés, ansiedad y depresión) (Accardo, 2017; Gelbar et al. et al., 2015; Jackson et al. et al., 2018). Además de los problemas sociales y académicos, debemos tener presente también que el acceso a la Universidad implica, en la mayoría de los casos, el acceso a la vida independiente con los desafíos que ello implica (Elias y White, 2018; Jackson et al. et al., 2018; Van Hees et al. et al., 2015; Vincent et al., 2017).

Recientemente, Hurley-Hanson y colaboradores (2020) han publicado un texto donde se reúne un conjunto de experiencias universitarias dirigidas explícitamente a personas con TEA. Estas experiencias son muy diversas, desde programas de empleo, programas de formación profesional, hasta apoyo para la obtención del grado universitario. De la misma forma que existe alguna universidad en USA especializada en estudiantes sordos (Gallaudet University, Washington DC D $^{3}$ NTID, National Technical Institute for the Deaf, Rochester, New York ${ }^{4}$; SWCID, Southwest Collegia-

https://www.gallaudet.edu/.

https://www.rit.edu/ntid/.

Ediciones Universidad de Salamanca / CC BY-NC-ND

Siglo Cero, vol. 52 (2), 2021, abril-junio, pp. 83-100 
te Institute for the Deaf, Big Spring, Texas ${ }^{5}$ ), han surgido en algunas universidades americanas programas de formación especializados en estudiantes que aprenden de forma diferente, como estudiantes con dislexia, Trastornos por Déficit de Atención e Hipeactividad o TEA (Landmarck College, Vermont ${ }^{6}$; Life College, Stanbridge University, California ${ }^{7}$, etc.). Es importante caer en la cuenta de la diferente legislación $y$, sobre todo, el concepto diferente de universidad existente entre las universidades americanas y europeas (en particular con las españolas). En este sentido, los programas que se recogen en estos centros no serían, en sentido estricto y en todos los casos, considerados programas de formación universitaria más allá de que se imparten en una institución con ese título.

\section{Programas de apoyo universitario}

De entre los programas de apoyo específicos para estudiantes universitarios que informan Hurley-Hanson y colaboradores (2020), destacamos los de la Universidad de Adelphi en Long Island ${ }^{8}$ (New York) y la Universidad Marshall de West Virgina'. También existen centros de investigación universitarios especializados en los TEA que ofrecen servicios para las personas con TEA de la comunidad. El Centro Burkhart de la Universidad de Texas ${ }^{10}$ o el Centro para el Autismo de la Universidad Ball State ${ }^{11}$ son ejemplos de ello.

Los modelos de intervención de los servicios de apoyo a estudiantes universitarios se han inspirado en el modelo ICIDH-2 (WHO, 2001). En este sentido, debemos tener en cuenta que muchos de los programas de intervención psicoeducativa, por ejemplo, en habilidades sociales, afrontamiento del estrés, etc., se enfocan generalmente en responsabilizar a las personas con TEA en cambiar (aprender a camuflarse en exposiciones sociales, aprender a relajarse, etc.). Por este motivo, debemos poner el acento en mejorar el ajuste entre la persona y el ambiente, modificando también el ambiente, en lugar de modificar solo a la persona (Mandy et al., 2016).

Del análisis de los programas de apoyo ofrecidos por las diferentes universidades consultadas, podemos concluir que los estudiantes con TEA se pueden beneficiar de prácticamente todas las medidas de apoyo desarrolladas para otros estudiantes con discapacidad (más tiempo para exámenes, situación privilegiada en el aula, ayuda para la toma de apuntes, etc.). No obstante, es importante resaltar la gran heterogeneidad e intensidad de los síntomas experimentados por las personas con TEA (Jeste y Geschwind, 2014). Como consecuencia de esta heterogeneidad y como consecuencia de la interacción con el entorno (falta de apoyos o apoyos inadecuados), los problemas

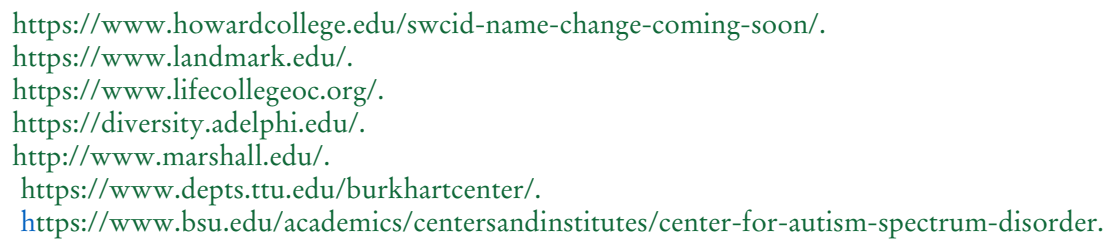


o situaciones de riesgo pueden variar. En definitiva, no todos los apoyos utilizados durante las etapas educativas previas o utilizados en el hogar tienen que ser útiles en la etapa de vida independiente y/o vida universitaria. Kettler (2012) propone que, antes de seleccionar e implementar los apoyos, es necesario indagar sobre las características personales del estudiante con TEA, en particular, en dos direcciones: la individual (comportamientos específicos TEA presentes en el estudiante) y la institucional (características ambientales del centro y título que desea cursar). Por ejemplo, es de destacar que la distribución de los estudiantes con TEA no es aleatoria en todos los títulos universitarios, produciéndose una cierta concentración en aquellos estudios más adaptados a sus características como son las áreas de Ciencias, Matemáticas, Tecnologías e Ingeniería (STEM) ${ }^{12}$ (Ehsan et al. et al., 2018; Wei et al. et al., 2013), lo cual no significa que no tengan dificultades en estas disciplinas. En todo caso, son estudios más propensos al trabajo individual y con menos contacto social. Este hecho nos debe hacer reflexionar, entre otras cosas, en el uso de los medios tecnológicos actuales como herramienta inclusiva en el aula (Alonso-Esteban y Alcantud-Marín, 2019).

\section{Apoyos específicos para los estudiantes universitarios con TEA}

Siguiendo el modelo de Alcantud (Alcantud-Marín, 1995; Alcantud-Marín et al. et al., 2000), organizaremos este apartado en tres grandes dimensiones: la social (programas de transición y acogida, programas de mentores y voluntarios), la dimensión técnica (curricular y recursos) y la dimensión personal (programas de entrenamiento en habilidades específicas).

\subsection{Intervención en dimensión social}

Como ya ha quedado expuesto, muchos estudiantes con TEA presentan dificultades en comunicación e interacción social a lo largo de toda su vida (Graetz, 2010). Estas dificultades les crearán grandes barreras para conseguir resultados académicos y sociales, asociándose con su calidad de vida, su empleabilidad, autoconfianza y, en general, con el desarrollo de habilidades personales (VanBergeijk et al. et al., 2008). Como todas las personas, un estudiante con TEA quiere tener amigos (relaciones con iguales), pero no sabe cómo desarrollar y mantener esa relación (Hendricks y Wehman, 2009). En muchas ocasiones, se forman grupos de iguales, todos ellos estudiantes con TEA, generándose pequeños grupos aislados o desarrollando actividades en solitario (Hendricks y Wehman, 2009). En este sentido, la mayoría de los servicios de apoyo no ofrecen apoyos específicos para incrementar la socialización (Adreon y Durocher, 2007). En la misma dirección, también tienen problemas a la hora de relacionarse con el profesorado, bien porque por su naturaleza inquisitiva, rigidez, dedicación, interés y curiosidad puedan poner en aprietos a los propios docentes, o bien

12 STEM del inglés: Science, Technology, Engineering and Mathematics. 
porque la falta de contacto visual o mirada huidiza hacen que el profesorado pueda interpretar un intento de engaño. Por ello, se hacen necesarios, también, programas de formación al profesorado sobre cómo interactuar con estudiantes con TEA. En definitiva, debemos empezar a pensar en la existencia de una "cultura del autismo", una forma de ser persona, con normas de comportamiento diferentes a las socialmente establecidas o aceptadas.

\subsection{Programas de sensibilización a la comunidad universitaria}

Es importante caer en la cuenta de que, cuando vemos un estudiante que utiliza el bastón blanco para guiarse por nuestras aulas, tendemos a ofrecerle el brazo para ayudarle o avisamos a otros estudiantes para que le dejen paso. Sin embargo, cuando un estudiante se dirige a nosotros con un lenguaje "extraño o idiosincrásico", con una mirada "huidiza", o simplemente rehúye el contacto visual ante nuestra presencia, tendemos a rechazarlo. En este sentido, creemos necesarias campañas informativas y formativas que nos lleven a no enjuiciar el comportamiento ajeno.

\subsection{Programas de transición}

Los entornos universitarios reúnen condiciones especiales y desconocidas para todos los estudiantes noveles. Sin embargo, la capacidad de adaptación de los estudiantes con TEA hace que los programas de transición y/o acogida sean apoyos específicos necesarios para ellos. De la misma forma que existen programas de transición de educación primaria a secundaria y bachiller (Mandy et al., 2016), algunas universidades españolas han desarrollado programas de transición a los estudios universitarios consistentes en visitas a los centros universitarios y orientación sobre los estudios. Sin embargo, estos programas suelen ser genéricos a toda la Universidad y están más relacionados con el proceso de acceso a la Universidad, las pruebas de selectividad, la toma de decisiones sobre cuál o cuáles estudios cursar, etc. Sin duda, estos momentos son de gran tensión para todos los estudiantes, más si cabe, para los estudiantes con TEA. La baja tolerancia a la frustración y las dificultades de autorregulación emocional pueden hacer necesaria una intervención preventiva o preparatoria. En USA existen programas preparatorios o de transición para estudiantes con TEA. Lei y colaboradores (2018) evaluaron un programa piloto que consistía en una escuela de verano para estudiantes con TEA, en la que los futuros estudiantes experimentaban diferentes contextos de la vida universitaria: académicos (asistencia a conferencias), sociales (grupos de iguales, actividades deportivas, lúdicas, etc.), administrativos (enfrentarse con la solicitud de plaza, tramitación de expedientes diversos, etc.) y de la vida diaria en la universidad (comer en el comedor universitario, residir en un colegio mayor, etc.). Los resultados de este programa apuntan hacia una reducción de las preocupaciones relacionadas con el acceso a la Universidad y su tránsito por ella. Ashbaugh y colaboradores (2017) desarrollan y evalúan un programa de intervención consistente en reuniones semanales en las que se aportaba un plan de actividades sociales alre- 
dedor de los intereses de los estudiantes con TEA. Los resultados de seguimiento demostraron que todos los participantes después de la intervención incrementaron el número de eventos sociales comunitarios y extracurriculares e interacciones con otros estudiantes, mejorando, también, su rendimiento académico y satisfacción personal.

Nuske y colaboradores (2019) desarrollan una revisión sistemática de las publicaciones en seis bases de datos, seleccionando solo 11 documentos que describen experiencias de transición a la educación superior desde la perspectiva de los estudiantes y sus familias. Es de destacar que todos ellos se han publicado en los últimos diez años.

Una experiencia española es la llevada a cabo en la Universidad Politécnica de Barcelona (Vilalta-Porta et al. et al., 2018), denominada Meetup, y en la que se trataba de desarrollar habilidades sociales, técnicas de estudio y planificación, tratamiento de la ansiedad y relaciones sociales. Debemos reflexionar sobre el hecho de que, de los siete estudiantes que iniciaron esta experiencia piloto en esta universidad, cuatro la abandonaron a los pocos días de su inicio. Los programas de transición deben evolucionar hacia programas de acogida individualizados para cada estudiante en su respectivo centro de estudios y respondiendo a sus necesidades específicas.

\subsection{Programas de tratamiento de la ansiedad}

La relación entre la ansiedad y los resultados académicos ha quedado ya demostrada (Sinha, 2014). Los tratamientos más eficaces para los trastornos de ansiedad no medicamentosos son los cognitivo-conductuales (Attwood, 2004). En el marco de estos programas de acogida o de forma independiente a ellos, se deben ofertar programas de intervención para la regulación de la ansiedad. Los trastornos por ansiedad son muy comunes entre las personas que desarrollan TEA. Hasta el $84 \%$ de ellos experimentan algún grado de ansiedad (White et al. et al., 2009), siendo una de las principales razones de consulta en los servicios de salud mental por parte de las personas con TEA y sus familias (Skokauskas y Gallagher, 2012). Los trastornos de ansiedad en las personas con TEA no siguen los patrones psicosociales de la manifestación de la ansiedad en personas neurotípicas, relacionándose con los síntomas nucleares de los TEA (Wood y Gadow, 2010). Entre las personas con TEA, las de alto funcionamiento son las que tienen mayor riesgo de presentar o desarrollar este trastorno (Davis et al., 2011; Van Steensel y Heeman, 2017). Fernández-Rodríguez y colaboradores (2019) realizan un estudio sobre una muestra de setecientos estudiantes universitarios de la Universidad de Oviedo en el que se midieron diferentes variables psicológicas. De este estudio, se desprende que un $44,7 \%$ presentaron malestar psicológico por ansiedad y el 13,5 \% manifestaron síntomas de depresión. Las situaciones que mayor fuente de tensión generaron fueron las de hablar en público, solicitud de ayuda en relación a sus problemas personales y las relacionadas con los hábitos de salud (sueño y alimentación). Dados estos resultados, se apunta la necesidad de la existencia de un programa específico de atención psicológica para todos los estudiantes $y$, en particular, aquellos que padecen alguna discapacidad (en el caso que nos 
ocupa, TEA). Estos datos han sido corroborados en varios estudios (Celis et al., 2001; Serrano-Barquin et al. et al., 2013).

\subsection{Programas de mentores y de pares voluntarios}

Los mentores son personas que hacen la función de guía o consejero sobre otra persona que se considera inexperta, protegida o aprendiz. En el caso de los estudiantes universitarios con TEA existen diferentes formatos. El mentor puede ser un profesional (docente o no) que orienta el estudio, da consejo al estudiante sobre las actividades universitarias y le asiste en caso de conflicto (Ames et al. et al., 2016).

En ocasiones, para facilitar la integración social se utiliza como mentores a compañeros iguales en el aula. Estas experiencias se han desarrollado fundamentalmente en aulas no universitarias (Gardiner e Iarocci, 2014), siendo uno de los recursos educativos (Programas de Instrucción mediados por pares) más efectivos (Zeneli y Tymms, 2015). Existen algunas experiencias universitarias para estudiantes con grandes necesidades apoyo (Carter et al. et al., 2005) y experiencias específicas para estudiantes universitarios con TEA (Haas et al. et al., 2019) en las que se intenta extrapolar las experiencias no universitarias. En general, reconociendo las bondades de la instrucción mediada por pares, las dificultades de encontrar estudiantes universitarios en la misma aula hacen difícil la sistematización de este tipo de apoyo.

\subsection{Programas de apoyo basados en el uso de tecnología}

Muchos estudiantes con TEA se matriculan de estudios relacionados con STEM debido a que el desarrollo de la actividad con la mediación de la tecnología les da algún tipo de seguridad. Ante las discusiones en grupo, muchos estudiantes prefieren las discusiones por correo electrónico o participación en foros electrónicos o blogs; frente a las discusiones en grupo, prefieren discusiones en tiempo real por medio de Skype u otro software para el desarrollo de reuniones (meeting). De esta forma evitan los desplazamientos hasta el aula, pero también muchos de ellos evitan la ansiedad producida por la interacción social. De la misma forma prefieren las tutorías mediadas por Skype a las presenciales y esto se extiende a todas las interacciones con el personal de administración y servicios de la Universidad (Satterfield et al. et al., 2015). El uso de sistemas de Blended Learning (Salinas et al. et al., 2018) como herramienta de instrucción en la Universidad no está restringido a los estudiantes con discapacidad (en particular con TEA) y puede considerarse una medida de inclusión (Alonso-Esteban y Alcantud-Marín, 2019).

\section{Conclusión}

Los servicios de apoyo universitarios a estudiantes con discapacidad se han desarrollado en los últimos 25-30 años. Desde la aparición de los mismos a finales de los 
años 80 del pasado siglo, se ha andado ya un largo camino, sobre todo en lo relacionado con la eliminación de barreras arquitectónicas en los edificios universitarios, medidas de accesibilidad electrónica y medidas de equiparación de oportunidades. Sin embargo, creemos que aún queda un largo camino hasta llegar a un campus universitario socialmente inclusivo. Aún existen reticencias y llama la atención que estudiantes con trastornos mentales, discapacidad intelectual o trastornos del espectro del autismo transiten por nuestras aulas. Son conocidas las experiencias de egresados con síndrome de Down, sin embargo, no se publicitan los éxitos de los estudiantes con síndrome de Asperger o trastornos del espectro del autismo y mucho menos los fracasos. El dato sobre la presencia de estudiantes con diferentes trastornos en la Universidad sigue siendo un dato oculto y reservado, como mucho, a las autoridades académicas.

En la revisión que se ha presentado en este artículo, es muy posible que se hayan quedado en el camino muchas otras experiencias, pero lo que se pretendía era dar a conocer la necesidad de desarrollar programas específicos para la atención de estos estudiantes y, quizás lo más importante, la necesidad de no hacer recaer sobre ellos (los estudiantes con trastornos del espectro del autismo) la responsabilidad exclusiva de su inclusión. No solo ellos deben aprender a regular sus emociones, sus fuentes de ansiedad y sus reacciones, es necesario también que nosotros aprendamos y participemos en el desarrollo de la cultura del autismo. El mayor conocimiento de los procesos de aprendizaje de las personas con TEA nos permitirá evolucionar y modificar nuestras prácticas, costumbres y nuestros entornos. Sin duda, la consecuencia de esta evolución será una Universidad mejor para todos.

\section{Referencias bibliográficas}

Accardo, A. (2017). College-bound young adults with ASD: self-reported factors promoting and inhibiting success. DADD Online Journal: Research to Practice, 4(1), 36-46. Recuperado el 23 de noviembre de 2019, de http://daddcec.org/Portals/O/CEC/Autism_Disabilities/Research/Publications/DOJ_Volume4_2017.pdf.

Adreon, D. y Durocher, J. (2007). Evaluating the college transition needs of individuals with high-functioning Autism Spectrum Disorders. Journal in School and Clinic, 42(5), 271-279. doi:10.1177/10534512070420050201

Aguilar-Pérez, L. (2011). Plan Bolonia: ventajas e inconvenientes para las personas con Síndrome de Asperger. En UNIDIS, Inclusión de los estudiantes con discapacidad en la universidad: necesidades y demandas (pp. 165-174). UNED. Recuperado el 12 de noviembre de 2019, de https://www.uned.es/universidad/dam/inicio/institucional/unidis/publicaciones/ publicaciones-unidis/documentos/Jornadas-Inclusion-Estudiantes-Discapacidad-Universidad.pdf.

Alcantud-Marín, F. (1995). Estudiantes con discapacidad integrados en los estudios universitarios: notas para su orientación. En F. Rivas, Manual de Asesoramiento y Orientación Vocacional (pp. 455-470). Síntesis.

Alcantud-Marín, F., Alonso-Esteban, Y. y Mata-Iturralde, S. (2016). Prevalencia de los Trastornos del Espectro Autista: revisión de datos. Siglo Cero, 47(4), 7-26. https:// doi:10.14201/scero2016474726 
ESTUDIANTES UNIVERSITARIOS CON TRASTORNOS DEL ESPECTRO DEL AUTISMO: REVISIÓN DE SUS NECESIDADES Y NOTAS PARA SU ATENCIÓN FRANCISCO ALCANTUD MARÍN Y YURENA ALONSO-ESTEBAN

Alcantud-Marín, F., Ávila-Clemente, V. y Asensi-Borras, M. (2000). La integración de estudiantes con dispacacidad en los estudios superiores. Valencia: Servei de Publicacions de la Universitat de Valencia. Recuperado el 2 de noviembre de 2019, de http://roderic. uv.es/handle/10550/22063.

Alonso-Esteban, Y. y Alcantud-Marín, F. (2019). Flipped class o blended learning como recurso para la inclusión de estudiantes con diversidad funcional en las aulas universitarias: aplicación piloto. En XIII Congreso Internacional de Educación e Innovación, Inclusión, Tecnologia y Sociedad. Dykinson.

Ames, M., McMorris, C., Alli, L. y Bebko, J. (2016). Overview and evaluation of a Mentorship Program for university students with ASD. Ocus on Autism and other Developmental Disabilities, 31(1), 27-36. https://doi:10.1177/1088357615583465

Anderson, A., Stephenson, J. y Carter, M. (2017). A systematic literature review of the experiences and supports of students with autism spectrum disorder in post-secondary education. Research in Autism Spectrum Disorders, 39, 33-53. https://doi:10.1016/j. rasd.2017.04.002

APA. (1994). Diagnostic and Statistical Manual of Mental Disorders. 4th Edition. American Psychiatric Association.

APA. (2013). Diagnostic and Statistical Manual of Mental Disorders DSM-5. American Psychiatric Association.

Ashbaugh, K., Koegel, R. y Koegel, L. (2017). Increasing social integration for college students with Autism Spectrum Disorder. Behavioral Development Bulletin, 22(1), 183-196. https://doi:10.1037/bdb0000057

AtTwood, T. (2004). Exploring feelings: anxiety: cognitive behaviour therapy to manage anxiety. Future Horizons.

Baio, J., Wiggins, L., Christensen, D., Maenner, M., Daniels, J., Warren, Z., ... y Dowling, N. (2018). Prevalence of Autism Spectrum Disorder among children aged 8 years - autism and developmental disabilities monitoring Network, 11 Sites, United States, 2014. MMWR Morbility and Mortality Weekly Report, 67(6), 1-23. Recuperado el 2 de mayo de 2018, de https://www.cdc.gov/mmwr/volumes/67/ss/ss6706a1.htm?s_cid=ss6706a1_w.

BELLINI, S. (2004). Social skill deficits and anxiety in high-functioning adolescents with autism spectrum disorders. Focus on Autism and Other Developmental Disabilities, 19(2), 78-86. https://doi:10.1177/10883576040190020201

Bellini, S. (2006). The development of social anxiety in adolescents with Autism Spectrum Disorders. Focus on Autism and Other Developmental Disabilities, 21(3), 138-145. https:// doi:10.1177/10883576060210030201

Berger, H., Aerts, F., Van Spaendonck, K., Cools, A. y Teunisse, J. (2003). Central coherence and cognitive shifting in relation to social improvement in high-functioning young adults with autism. Journal of Clinical and Experimental Neuropsychology, 25(4), 502-511. https://doi:10.1076/Jcen.25.4.502.13870

Billstedt, E., Gillberg, I. y Gillberg, C. (2007). Autism in adults: symptom patterns and early childhood predictors. Use of the DISCO-10 in a community sample followed from childhood. Journal of Child Psychology and Psychiatry, 48(11), 1102-1110. https:// doi:10.1111/j.1469-7610.2007.01774.x

BOE. (2003). Ley de Igualdad de Oportunidades, No Discriminación y Accesibilidad Universal para las personas con Discapacidad. Boletín Oficial del Estado, 289, 43197-43195.

BOE. (2007). Ley Orgánica 4/2007, de 12 de abril. Boletín Oficial del Estado, 89, 16241-16260.

Ediciones Universidad de Salamanca / CC BY-NC-ND

Siglo Cero, vol. 52 (2), 2021, abril-junio, pp. 83-100 
Braun, K., Christensen, D., Doernberg, N., Schieve, L., Rice, C., Wiggins, L., ... y YearGIN-Allsopp, M. (2015). Trends in the prevalence of autism spectrum disorder, cerebral palsy, hearing loss, intellectual disability and vision impairmente metropolitan Atlanta 1991-2010. PLos ONE, 10(4), e0124120.

Canal, R., García, P., Martín, M., Santos, J., Guisuraga, Z., Herráez, L., ... y Posada, M. (2010). Modified checklist for autism in toddlers: cross-culttural adaptation and validation in Spain. Journal of Autism and Developmental Disorders, 41, 1342-1351. https:// doi:10.1007/s10803-010-1163-z

Carter, E., Cushing, L., Clark, N. y Kennedy, C. (2005). Effects of peer support interventions on students' access to the general curriculum and social interactions. Research and Practice for Persons with Severe Disabilities, 30(1), 15-25. https://doi:10.2511/rpsd.30.1.15

Celis, J., Bustamante, M., Cabrera, D., Cabrera, M., Alarcón, W. y Monge, E. (2001). Ansiedad y estrés académico en estudiantes de medicina humana del primer y sexto año. Anales de la Facultad de Medicina, 62(1), 25-30. Recuperado el 5 de noviembre de 2019, de https://www.redalyc.org/pdf/379/37962105.pdf.

Davis, E., Moree, B., Dempsey, T., Reuther, E., Fodstad, J., Hess, J., ... y Matson, J. (2011). The relationship between autism spectrum disorders and anxiety. Research in Autism Spectrum Disorders, 5(1), 324-329. https://doi:10.1016/j.rasd.2010.04.015

De la Fuente-Anuncibay, R. y Cuesta-Gómez, J. (2017). Inclusión de alumnado con trastorno del espectro del autismo en la universidad: análisis y respuestas desde una dimensión internacional. International Journal of Developmental and Educational Psychology, 1(3), 13-22. https://doi:10.17060/ijodaep.2017.n1.v4.1023

Ehsan, H., Rispoli, M., Lory, C. y Gregori, E. (2018). A systematic review of STEM instruction with students with Autism Spectrum Disorders. Journal of Autism ans Developmental Disorders, 5(4), 327-348. https://doi:10.1007/s40489-018-0142-8

Elias, R. y White, S. (2018). Autism goes to college: understanding the needs of a student population on the Rise. Journal of Autism and Developmental Disorders, 48(3), 732-746. https://doi:10.1007/s10803-017-3075-7

Fernández-Rodríguez, C., Soto-López, T. y Cuesta, M. (2019). Needs and demands for psychological care in university students. Psicothema, 31(4), 414-421. https://doi:10.7334/ psicothema2019.78

Fombonne, E. (2009). Epidemiology of perversive developmental disorders. Pediatric Research, 65(6), 591-598.

Fortea, M., Escandell, M. y Castro, J. (2013). Estimación de la prevalencia de los Trastornos del Espectro Autista en Canarias. Anales de Pediatría, 79(6), 352-359. https//dx.doi. org/10.1016/j.anpedi.2013.04.022

Gardiner, E. e Iarocci, G. (2014). Students with Autism Spectrum Disorder in the university context: peer acceptance predicts intention to volunteer. Journal of Autism and Developmental Disorders, 44( 5), 1008-1017. https://doi:10.1007/s10803-013-1950-4

Gelbar, N., Shefgyk, A. y Reichow, B. (2015). A comprehensive survey of current and former college students with Autism Spectrum Disorders. Yale Journal of Biology and Medicine, 88(1), 45-68. Recuperado el 23 de noviembre de 2019, de https://www.ncbi.nlm.nih. gov/pmc/articles/PMC4345538/pdf/yjbm_88_1_45.pdf.

Gelbar, N., Smith, I. y Reichow, B. (2014). Systematic review of articles describing experience and supports of individuals with autism enrolled in college and university programs.

Ediciones Universidad de Salamanca / CC BY-NC-ND

Siglo Cero, vol. 52 (2), 2021, abril-junio, pp. 83-100 
ESTUDIANTES UNIVERSITARIOS CON TRASTORNOS DEL ESPECTRO DEL AUTISMO: REVISIÓN DE SUS NECESIDADES Y NOTAS PARA SU ATENCIÓN FRANCISCO ALCANTUD MARÍN Y YURENA ALONSO-ESTEBAN

Journal of Autism and Developmental Disorders, 44(10), 2593-2601. https://doi:10.1007/ s10803-014-2135-5

Graetz, J. (2010). Autism grows up: opportunities for adults with autism. Journal of Disability and Society, 25(1), 33-47. https://doi:10.1080/09687590903363324

Grogan, G. (2015). Supporting students with autism in higher education through teacher educator programs. SRATE Journal, 24(2), 8-13. Recuperado el 23 de noviembre de 2019, de https://files.eric.ed.gov/fulltext/EJ1083126.pdf.

Gurbuz, E., Hanley, M. y Riby, D. (2019). University students with autism: the social and academic experiences of university in the UK. Journal of Autism and Developmental Disorders, 49(2), 617-631. https://doi:10.1007/s10803-018-3741-4

HaAs, A., Vannest, K. y Smith, S. (2019). Utilizing peers to support academic learning for children with Autism Spectrum Disorder. Behavior Analysis in Practice, 12(3), 734-740. https://doi:10.1007/s40617-019-00363-4

Harpur, J., Lawlor, M. y Fitzgerald, M. (2004). Succeedind in college with Asperger Syndrome: a student guide. Jessica Kingsley Publichers.

Helt, M., Kelley, E., Kinsbourne, M., Pandey, J., Boorstein, H., Herbert, M. y Fein, D. (2008). Can children with autism recover? Is so, how? Neuropsychology Review, 18, 339366.

Hendricks, D. y Wehman, P. (2009). Transition from school to adulthood for youth with Autism Spectrum Disorders: review and recommendations. Focus on Autism and other Developmental Disabilities, 24(2), 77-88. https://doi:10.1177/1088357608329827

Hurley-Hanson, A., Giannantonio, C. y Griffiths, A. (2020). Universities with Autism Initiatives. En A. Hurley-Hanson, C. Giannantonio y A. Griffiths, Autism in the workplace: creating positive employment and career outcomes for generation A (pp. 111125). Palgrave Macmillan. https://doi:10.1007/978-3-030-29049-8_6

Jackson, S., Hart, L., Brown, J. y Volkmar, F. (2018). Brief report: self-reported academic, social, and mental health experiences of post-secondary students with autism spectrum disorder. Journal of Autism and Developmental Disorders, 48(3), 643-650. https:// doi:10.1007/s10803-017-3315-x

Jansen, D., Petry, K., Ceulemans, E., Noens, I. y Baeyens, D. (2017). Functioning and participation problems of students with ASD in higher education: which reasonable accommodations are effective? European Journal of Special Needs Education, 32(1), 71-88. https://doi: 10.1080/08856257.2016.1254962

Jeste, S. y Geschwind, D. (2014). Disentangling the heterogeneity of autism spectrum disorder through genetic findings. Nature Reviews Neurology, 10, 74-81. https://doi:10.1038/ nrneurol.2013.278

KetTlen, R. (2012). Testing accommodations: theory and research to inform practice. International Journal of Disability, Development and Education, 59(1), 43-66. https://doi:10.10 80/1034912X.2012.654952

King, M. y Bearman, P. (2009). Diagnostic change and the increased prevalence of autism. International Journal of Epidemiology, 38(5), 1224-1234.

Lei, J., Calley, S., Brosnan, M., Ashwin, C. y Russell, A. (2018). Evaluation of a transition to university programme for students with Autism Spectrum Disorder. Journal of Autism and Developmental Disorders, 1-15. https://doi:10.1007/s10803-018-3776-6

Mandy, W., Murin, M., Baykaner, O., Staunton, S., Сobb, R., Hellriegel, J., ... у Skuse, D. (2016). Easing the transition to secondary education for children with autism spectrum disorder: an evaluation of the systemic transition in education program- 
ESTUDIANTES UNIVERSITARIOS CON TRASTORNOS DEL ESPECTRO DEL AUTISMO: REVISIÓN DE SUS NECESIDADES Y NOTAS PARA SU ATENCIÓN FRANCISCO ALCANTUD MARÍN Y YURENA ALONSO-ESTEBAN

me for Autism Spectrum Disorder (STEP-ASD). Autism, 20(5), 580-590. https://doi: 10.1177/1362361315598892

Morales-Hidalgo, P., Roigé-Castellvi, J., Hernández-Martínez, C., Voltas, N. y CaNALS, J. (2018). Prevalence and characteristics of Autism Spectrum Disorder among Spanish school-age children. Journal of Autism and Developmental Disorders, 48(9), 3176-3190. https://doi:10.1007/s10803-018-3581-2

Newman, L., Wagner, M., Knokey, A., Marder, C., Nagle, K., Shaver, D. y Wei, X. (2011). The post-high school outcomes of young adults with disabilities up to 8 years after high school. IES Institute of Education Sciences (National Center for Special Education Research) U.S. Departament of Education. Recuperado el 5 de noviembre de 2019, de https:// nlts2.sri.com/reports/2011_09_02/

Nuske, A., Rillotta, F., Bellon, M. y Richdale, A. (2019). Transition to Higher Education for Students With Autism: a systematic literature review. Journal of Diversity in Higher Education. https://doi:10.1037/dhe0000108

Orsmond, G., Shatuck, P., Cooper, B., Sterzing, P. y Anderson, K. (2013). Social participation among young adults with an Autism Spectrum Disorder. Journal of Autism and Developmental Disorders, 43(11), 2710-2719. https://doi:10.1007/s10803-013-1833-8

Pérez-Crespo, L., Prats-Uribe, A., Tobias, A., Duran-Taulería, E., Coronado, R., Hervás, A. y Guxens, M. (2019). Temporal and geographical variability of prevalence and incidence of Autism Spectrum Disorder diagnoses in children in Catalonia, Spain. Autism Research, 12(11), 1693-1705. https://doi:10.1002/aur.2172

Ritvo, R., Ritvo, E., Guthrie, D., Ritvo, M., Hufnagel, D., McMahon, D., ... y Eloff, J. (2011). The Ritvo Autism Asperger Diagnostic Scale-Revised (RAADS-R): a scale to assist the diagnosis of Autism Spectrum Disorder in adults: an international validation study. Journal of Autism and Developmental Disorders, 41(8), 1076-1089. https://doi:10.1007/ s10803-010-1133-5

Rutter, M. (1970). Autistic children: infancy to adulthood. Seminars in Psychiatry, 2, 435-450.

Salinas, J., De Benito, B., Pérez, A. y Gisbert, M. (2018). Blended learning, más allá de la clase presencial. RIED Revista Iberoamericana de Educación a Distancia, 21(1), 195-213. https://doi:10.5944/ried.21.1.18859

SARretT, J. (2018). Autism and accommodations in higher education: insights from the autism community. Journal of Autism and Developmental Disorders, 48(3), 679-693. https:// doi:10.1007/s10803-017-3353-4

Satterfield, D., Lepage, C. y Ladjahasan, N. (2015). Preferences for online course delivery methods in higher education for students with autism spectrum disorders. Procedia Manufacturing, 3, 3651-3656. https://doi:10.1016/j.promfg.2015.07.758

Serrano-Barquin, C., Rojas-García, A. y Ruggero, C. (2013). Depresión, ansiedad y rendimiento académico en estudiantes universitarios. Revista Intercontinental de Psicología y Educación, 15(1), 47-60. Recuperado el 2 de noviembre de 2019, de https://www.redalyc. org/articulo.oa?id=80225697004.

ShatTuck, P. (2006). The contribution of diagnostic substitution to the growing administrative prevalence of autism in US special education. Pediatrics, 117(4), 1028-1037.

Sigman, M., Ruskin, E., Arbeile, S., Corona, R., Dissanayake, C., Espinosa, M. y Zierhut, C. (1999). Continuity and change in the social competence of children with autism, Down Syndrome, and developmental delays. Monographs of the Society for Research in Childs Development, 64, 1-114.

Ediciones Universidad de Salamanca / CC BY-NC-ND

Siglo Cero, vol. 52 (2), 2021, abril-junio, pp. 83-100 
ESTUDIANTES UNIVERSITARIOS CON TRASTORNOS DEL ESPECTRO DEL AUTISMO:

REVISIÓN DE SUS NECESIDADES Y NOTAS PARA SU ATENCIÓN

FRANCISCO ALCANTUD MARÍN Y YURENA ALONSO-ESTEBAN

SinHA, A. (2014). Stress vs academic performance. SCMS Journal of Indian Management, 11(4), 46. Recuperado el 17 de noviembre de 2019, de https://www.coursehero.com/ file/41645724/Stress-vs-Academic-Performancepdf/.

Skokauskas, N. y Gallagher, L. (2012). Mental health aspects of autistic spectrum disorders in children. Journal of Intellectual Disability Research, 36(3), 248-257. https://doi:10.1111/ j.1365-2788.2011.01423.x

UnIVERsia. (2016). Universidad y discapacidad: III estudio sobre el grado de inclusión del sistema universitario español respecto de la realidd de la discapacidad. Madrid: Fundación Universia. Recuperado el 25 de noviembre de 2019, de https://www.fundacionuniversia. net/wp-content/uploads/2017/02/Fundacion_IIIEstudio_digital_accesible.pdf.

Universia. (2018). Universidad y discapacidad. IV estudio sobre el grado de inclusión del sistema universitario español respecto de la realidad de la discapacidad. Madrid: Fundación Universia. Recuperado el 12 de noviembre de 2019, de https://www.fundacionuniversia. net/iv-estudio-universidad-y-discapacidad/.

Valdunquillo-Carlón, M. e Iglesias-Rodríguez, A. (2015). Una puerta abierta a la inclusión en la Universidad: experiencia con un alumno Erasmus con Síndrome de Asperger. Revista de Educación Inclusiva, 8(1), 104-114. Recuperado el 10 de octubre de 2019, de https://www.revistaeducacioninclusiva.es/index.php/REI/article/view/123/118.

Van Hees, V., Moyson, T. y Roeyers, H. (2015). Higher education experiences of students with autism spectrum disorder: challenges, benefits and support needs. Journal of Autism and Developmental Disorders, 45(6), 1673-1688. https://doi:10.1007/s10803-014-2324-2

Van Steensel, F. y Heeman, E. (2017). Anxiety levels in children with Autism Spectrum Disorder: a meta-analysis. Journal of Child and Family Studies, 26(7), 1753-1767. https:// doi:10.1007/s10826-017-0687-7

Vanbergeijk, E., Klin, A. y Volkmar, F. (2008). Supporting more able students on the autism spectrum: college and beyond. Journal of Autism and Developmental Disorders, 38(7), 1359-1370. https://doi:10.1007/s10803-007-0524-8

Vilalta-Porta, M., López-Álvarez, D., Montero-Mercadé, L., Mampel-Caballero, S. y Gracia-Blanes, M. (2018). Meetup de inclusión: programa de orientación para estudiantes con Trastorno de Espectro Autista (TEA) de la Universidad Politècnica de Catalunya (UPC). En Libro de Actas IV Congreso Universidad y Discapacidad (pp. 54-65). Fundación ONCE. Recuperado el 16 de noviembre de 2019, de https://ciud.fundaciononce.es/sites/ default/files/libro-de-actas-iv-congreso-universidad-y-discapacidad.pdf.

Vincent, J., Potts, M., Fletcher, D., Hodges, S., Howells, J., Mitchell, A., ... y Ledger, T. (2017). 'I think autism is like running on Windows while everyone else is a Mac': using a participatory action research approach with students on the autistic spectrum to rearticulate autism and the lived experience of university. Journal of Educational Action Research, 25(2), 300-315. https://doi:10.1080/09650792.2016.1153978

Watkins, L., Ledbetter-Cho, K., O’Reilly, M., Barnard-Brak, L. y García-Grau, P. (2019). Interventions for students with autism in inclusive settings: a best-evidence synthesis and meta-analysis. Psychological Bulletin, 145(5), 490-507. https://doi:10.1037/bul0000190

Wei, X., Wagner, M., Hudson, L., Yu, J. y Javitz, H. (2016). The Effect of transition planning participation and goal-setting on college enrollment among youth with Autism Spectrum Disorders. Remedial and Special Education, 37(1), 3-14. https://doi:10.1177/0741932515581495

Wei, X., Yu, J., Shattuck, P., McCraken, M. y Blackorby, J. (2013). Science, technology, engineering, and mathematics (STEM) participation among college students with an

Ediciones Universidad de Salamanca / CC BY-NC-ND

Siglo Cero, vol. 52 (2), 2021, abril-junio, pp. 83-100 
Autism Spectrum Disorder. Journal of Autism and Developmental Disorders, 43(7), 15391546. https://doi:10.1007/s10803-012-1700-z

White, S., Ollendick, T. y Bray, B. (2011). College students on the autism spectrum: prevalence and associated problems. Autism, 15(6), 683-701. https://doi:10.1177/1362361310393363

White, S., Oswald, D., Ollendick, T. y Scahill, L. (2009). Anxiety in children and adolescents with autism spectrum disorders. Clinical Psychology Review, 29(3), 216-229. https:// doi:10.1016/j.cpr.2009.01.003

WHO. (2001). International Classification of Functioning, Disability and Health ICIDH-2. World Health Organization.

Wood, J. y GADOw, K. (2010). Exploring the Nature and function of anxiety in youth. Journal of Clinical Psychology, 17(4), 281-292. https://doi:10.1111/j.1468-2850.2010.01220.x

Zeneli, M. y Tymms, P. (2015). A review of peer tutoring interventions and social interdependence characteristics. International Journal for Cross-Disciplinary Subjects in Education, 5(2), 2504-2510. https://doi:10.20533/ijcdse.2042.6364.2015.0341

Zürcher, N., Donnelly, N., Rogier, O., Russo, B., Hippolyte, L., Hadwin, J., ... y HadJIKHANI, N. (2013). It's all in the eyes: subcortical and cortical activation during grotesqueness perception in autism. PloSone, 8(1), 1-14. https://doi:10.1371/journal.pone.0054313 